\title{
On Approach to Optimize Manufacturing of a Four Stages Distributed Amplifier to Increase Density of Their Elements Under Influence of Miss-match Induced Stress and Porosity of Materials on Technological Process
}

Evgeny L. Pankratov ( $\sim$ elp2004@mail.ru )

Nizhny Novgorod State University

\section{Research Article}

Keywords: four stages distributed amplifier, optimization of manufacturing, increasing of density of elements, analytical approaches of modelling

Posted Date: January 17th, 2022

DOI: https://doi.org/10.21203/rs.3.rs-1197911/v1

License: (c) (1) This work is licensed under a Creative Commons Attribution 4.0 International License. Read Full License 


\title{
On approach to optimize manufacturing of a four stages distributed amplifier to increase density of their elements under influence of miss- match induced stress and porosity of materials on technological pro-
}

cess

\author{
E.L. Pankratov
}

1. Nizhny Novgorod State University, 23 Gagarin avenue, Nizhny Novgorod, 603950, Russia

2. Nizhny Novgorod State Technical University, 24 Minin Street, Nizhny Novgorod, 603950, Russia

elp2004@mail.ru

\begin{abstract}
We consider a way to obtain larger density of field-effect transistors, which includes into itself a four stages distributed amplifier. To describe the way we will consider fabrication the above amplifier in the framework of a heterostructure, which has a specifical structure. Some specific areas of the considered heterostructure must be doped by ion implantation or by diffusion. After the doping the considered dopant and radiation defects, which were generated during ion implantation, must be annealed by using considered in this paper optimized procedure. We also introduced a way for reducing of value of mismatch-induced stress in the framework of the considered heterostructure. It has been also introduced an analytical methodology for analysis of mass transport in the framework of the heterostructures during production of the considered type of integrated circuits. The methodology gives a possibility to take into account mismatch-induced stress.

Keywords: four stages distributed amplifier; optimization of manufacturing; increasing of density of elements; analytical approaches of modelling.

\section{Introduction}

Currently some questions of electronics of the solid state are elaborating [1-6]. Increasing of performance could be obtained by choosing materials, which have larger mobility of carriers of charge [7-10]. Reduction of dimensions of elements of microcircuits could be obtained in heterostructures with thin films [3-5,11] with using optimization of doping [12] and improvement of materials of layers [13-15]. Reduction of size of the above elements could be also used due to microwave or laser types of
\end{abstract}


annealing [16-18]. In the present paper we consider a way to fabricate field-effect heterotransistors in a four stages distributed amplifier with smaller size and smaller mismatch-induced stress. The amplifier was fabricated in a heterostructure, which includes into itself epitaxial layer with several specific sections (other materials were used), which was grown on a porous buffer layer. The buffer layer was grown on a substrate (see Fig. 1). A dopant should be infused or implanted into the considered sections in the epitaxial layer to produce type of conductivity ( $p$ or $n$ ), which required to fabricate recently considered transistors. These sections after doping will be drains, sources and gates (see Fig. 1). Main aim of this paper is formulation of conditions to reduce of the considered amplifier's elements with increasing of their elements and reduction of mismatch-induced stress.

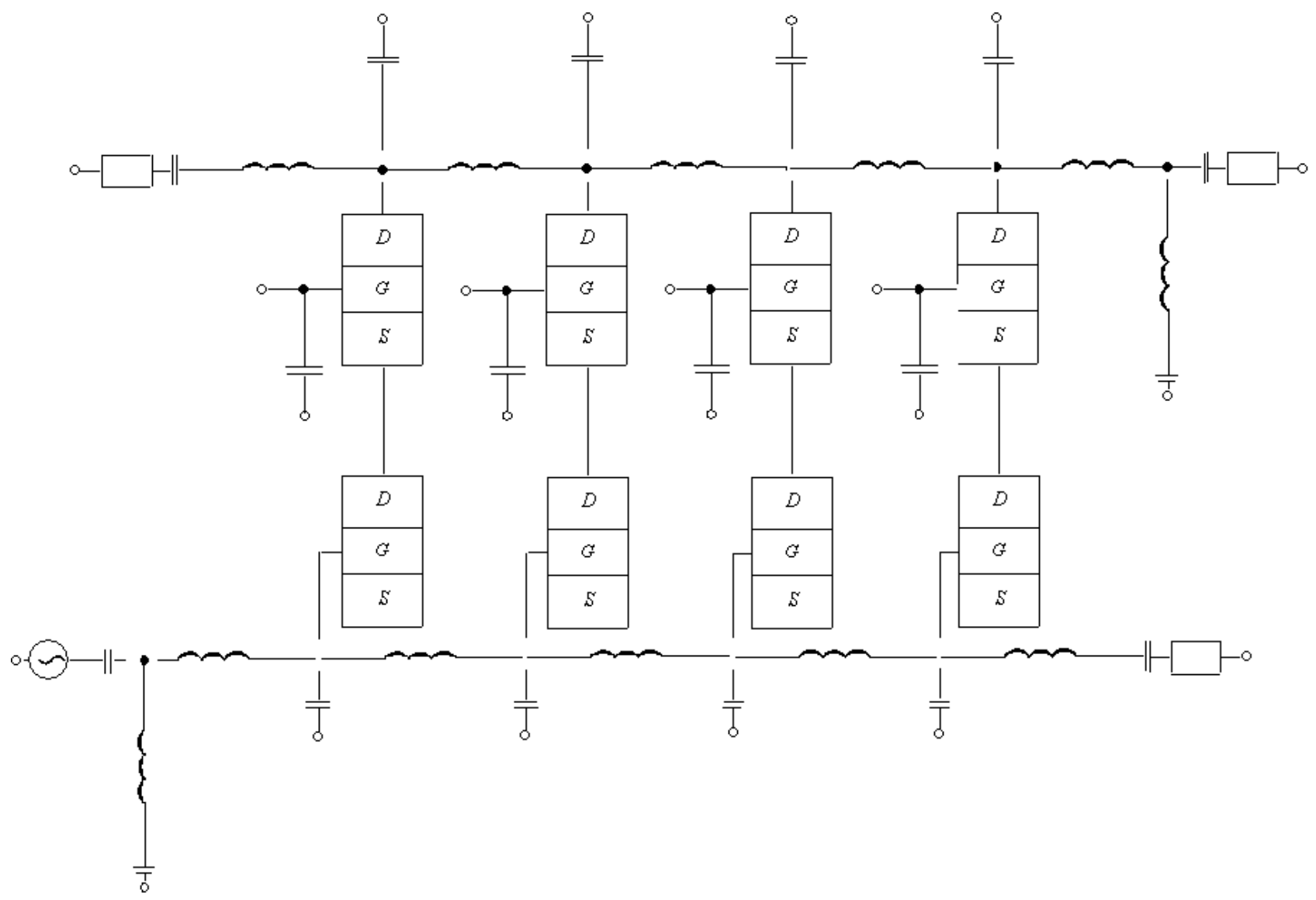

Fig. 1a. Structure of the considered amplifier [19] 


\section{Epitaxial layer}

\section{Buffer layer}

Substrate

Fig. 1b. Epitaxial layer, buffer layer and substrate in the considered heterostructure (view from side)

\section{Method of solution}

We will analyze distribution of concentration of dopant in space and time in the heterostructure from Fig. 1 by solving the second law of Fick [1,20-23]

$$
\begin{aligned}
& \frac{\partial C(x, y, z, t)}{\partial t}=\frac{\partial}{\partial x}\left[D \frac{\partial C(x, y, z, t)}{\partial x}\right]+\frac{\partial}{\partial y}\left[D \frac{\partial C(x, y, z, t)}{\partial y}\right]+\frac{\partial}{\partial z}\left[D \frac{\partial C(x, y, z, t)}{\partial z}\right]+ \\
& +\Omega \frac{\partial}{\partial x}\left[\frac{D_{S}}{k T} \nabla_{S} \mu_{1}(x, y, z, t) \int_{0}^{L_{z}} C(x, y, W, t) d W\right]+ \\
& +\Omega \frac{\partial}{\partial y}\left[\frac{D_{S}}{k T} \nabla_{S} \mu_{1}(x, y, z, t) \int_{0}^{L_{z}} C(x, y, W, t) d W\right]+(1) \\
& +\frac{\partial}{\partial x}\left[\frac{D_{C S}}{\bar{V} k T} \frac{\partial \mu_{2}(x, y, z, t)}{\partial x}\right]+\frac{\partial}{\partial y}\left[\frac{D_{C S}}{\bar{V} k T} \frac{\partial \mu_{2}(x, y, z, t)}{\partial y}\right]+\frac{\partial}{\partial z}\left[\frac{D_{C S}}{\bar{V} k T} \frac{\partial \mu_{2}(x, y, z, t)}{\partial z}\right] .
\end{aligned}
$$

Initial and boundary conditions for the Eq. (1) could be written as

$$
\begin{gathered}
\left.\frac{\partial C(x, y, z, t)}{\partial x}\right|_{x=0}=0,\left.\frac{\partial C(x, y, z, t)}{\partial x}\right|_{x=L_{x}}=0,\left.\frac{\partial C(x, y, z, t)}{\partial y}\right|_{y=0}=0, C(x, y, z, 0)=f_{C}(x, y, z), \\
\left.\frac{\partial C(x, y, z, t)}{\partial y}\right|_{x=L_{y}}=0,\left.\frac{\partial C(x, y, z, t)}{\partial z}\right|_{z=0}=0,\left.\frac{\partial C(x, y, z, t)}{\partial z}\right|_{x=L_{z}}=0 .
\end{gathered}
$$


Function $C(x, y, z, t)$ describes distribution of dopant's concentration in space and time; $x, y$ and $z$ are the spatial coordinates; $t$ is the current time; parameter $\Omega$ describes value of volume of atomic of dopant; symbol $\nabla_{s}$ describes operator of surficial gradient; integral $\int_{0}^{L_{z}} C(x, y, z, t) d z$ describes dopant's surficial concentration on the interface between heterostructure's layers (we used Z-axis as a perpendicular to interface between heterostructure's layers); functions $\mu_{1}(x, y, z, t)$ and $\mu_{2}(x, y, z, t)$ describe chemical potential for two cases: accounting of mismatch-induced stress and accounting of material's porosity; parameters $D$ and $D_{S}$ describe volumetric diffusion coefficient and surficial diffusion coefficient, respectively. The above coefficients will be changed with changing of materials of heterostructure. They will be also changed with changing of cooling and heating rates when dopant and/or radiation defects are annealing. Variations of distributions of dopant's and radiation defect's concentrations in space and time also changing values of dopant diffusion coefficients. Based on empirical data [24-26] dependences of dopant diffusion coefficients on parameters are following

$$
\begin{aligned}
& D_{C}=D_{L}(x, y, z, T)\left[1+\xi \frac{C^{\gamma}(x, y, z, t)}{P^{\gamma}(x, y, z, T)}\right]\left[1+\varsigma_{1} \frac{V(x, y, z, t)}{V^{*}}+\varsigma_{2} \frac{V^{2}(x, y, z, t)}{\left(V^{*}\right)^{2}}\right], \\
& D_{S}=D_{S L}(x, y, z, T)\left[1+\xi_{S} \frac{C^{\gamma}(x, y, z, t)}{P^{\gamma}(x, y, z, T)}\right]\left[1+\varsigma_{1} \frac{V(x, y, z, t)}{V^{*}}+\varsigma_{2} \frac{V^{2}(x, y, z, t)}{\left(V^{*}\right)^{2}}\right] .
\end{aligned}
$$

Functions $D_{L}(x, y, z, T)$ and $D_{L S}(x, y, z, T)$ describe dependences of dopant diffusion coefficients on spatial coordinates and temperature $T$; function $P(x, y, z, T)$ describes the dopant's limit of solubility; value of parameter $\gamma$ depends on material's condition and could be integer usually in interval $\gamma \in[1,3]$ [24]; function $V(x, y, z, t)$ describes distribution of radiation vacancies' concentration in space and time distribution in the equilibrium state $V^{*}$. Dependence of diffusion coefficient of dopant on concentration of the dopant was described in [24]. Distributions of point radiation defects concentrations were calculated as solution of the system of the second Fick's laws [20-23,25,26] 


$$
\begin{gathered}
\frac{\partial \rho(x, y, z, t)}{\partial t}=\frac{\partial}{\partial x}\left[D_{\rho}(x, y, z, T) \frac{\partial \rho(x, y, z, t)}{\partial x}\right]+\frac{\partial}{\partial y}\left[D_{\rho}(x, y, z, T) \frac{\partial \rho(x, y, z, t)}{\partial y}\right]+ \\
+\frac{\partial}{\partial z}\left[D_{\rho}(x, y, z, T) \frac{\partial \rho(x, y, z, t)}{\partial z}\right]-k_{\rho, \rho}(x, y, z, T) \rho^{2}(x, y, z, t)-k_{t, V}(x, y, z, T) \times \\
\times I(x, y, z, t) V(x, y, z, t)+\Omega \frac{\partial}{\partial x}\left[\frac{D_{\rho S}}{k T} \nabla_{s} \mu(x, y, z, t) \int_{0}^{L_{z}} \rho(x, y, W, t) d W\right]+ \\
+\Omega \frac{\partial}{\partial y}\left[\frac{D_{\rho S}}{k T} \nabla_{s} \mu(x, y, z, t) \int_{0}^{L_{z}} \rho(x, y, W, t) d W\right]+\frac{\partial}{\partial x}\left[\frac{D_{\rho S}}{\bar{V} k T} \frac{\partial \mu_{2}(x, y, z, t)}{\partial x}\right]+ \\
+\frac{\partial}{\partial y}\left[\frac{D_{\rho s}}{\bar{V} k T} \frac{\partial \mu_{2}(x, y, z, t)}{\partial y}\right]+\frac{\partial}{\partial z}\left[\frac{D_{\rho S}}{\bar{V} k T} \frac{\partial \mu_{2}(x, y, z, t)}{\partial z}\right] .
\end{gathered}
$$

Initial and boundary conditions could be written as

$$
\begin{gathered}
\left.\frac{\partial \rho(x, y, z, t)}{\partial x}\right|_{x=0}=0,\left.\frac{\partial \rho(x, y, z, t)}{\partial x}\right|_{x=L_{x}}=0,\left.\frac{\partial \rho(x, y, z, t)}{\partial y}\right|_{y=0}=0, \\
\left.\frac{\partial \rho(x, y, z, t)}{\partial y}\right|_{y=L_{y}}=0,\left.\frac{\partial \rho(x, y, z, t)}{\partial z}\right|_{z=0}=0,\left.\frac{\partial \rho(x, y, z, t)}{\partial z}\right|_{z=L_{z}}=0, \\
\rho(x, y, z, 0)=f_{V}(x, y, z), V\left(x_{1}+V_{n} t, y_{1}+V_{n} t, z_{1}+V_{n} t, t\right)=V_{\infty}\left(1+2 \lambda \omega / k T \sqrt{x_{1}^{2}+y_{1}^{2}+z_{1}^{2}}\right) .
\end{gathered}
$$

Parameter $\rho$ is equal to $I$ (in the second Fick's law for interstitials) or $V$ (in the second Fick's law for vacancies); function $I(x, y, z, t)$ describes distribution of radiation interstitials' concentration in space and time distribution in the equilibrium state $I^{*}$; functions $D_{\rho}(x, y, z, T)$ describe volumetric diffusion coefficients of point radiation defects; functions $D_{\rho S}(x, y, z, T)$ describe surficial diffusion coefficients of point radiation defects; terms $V^{2}(x, y, z, t)$ and $I^{2}(x, y, z, t)$ in Eqs. (3) describe generation simplest complexes of point radiation defects (i.e. divacancies and diinterstitials) [26]; function $k_{I, V}(x, y, z, T)$ describes dependences of the parameter of point radiation defects recombination on spatial coordinates and temperature; function $k_{\rho, \rho}(x, y, z, T)$ describe dependences of the parameters generation of complexes of point radiation defects on spatial 
coordinates and temperature; parameter $k$ describes the constant of Boltzmann; parameter $\omega$ is equal to $a^{3}$, where $a$ is the interatomic distance; parameter $\lambda$ describes the specific surface energy. To take into account modification of porosity of material of buffer layers we assume, that porous have approximately cylindrical form with average size $r=\sqrt{x_{1}^{2}+y_{1}^{2}}$ and $z_{1}$ before starting of annealing [23]. During annealing small pores will decomposing on several vacancies. These vacancies will be absorbed by larger pores in the porous layer [27]. At the same time the larger pores will be more spherical [27]. Distribution of vacancies' concentration in the considered heterostructure, which are exist due to porosity of materials of heterostructure, could be calculated as a sum of all pores, i.e.

$$
V(x, y, z, t)=\sum_{i=0}^{l} \sum_{j=0}^{m} \sum_{k=0}^{n} V_{p}(x+i \alpha, y+j \beta, z+k \chi, t), R=\sqrt{x^{2}+y^{2}+z^{2}} .
$$

Parameters $\alpha, \beta$ and $\chi$ describe the averaged lengths between centers of pores in recently considered directions $x, y$ and $z$; parameters $l, m$ and $n$ are equal to quantities of pores in the considered directions.

We calculate distributions of concentrations of diinterstitials $\Phi_{I}(x, y, z, t)$ and divacancies $\Phi_{V}(x, y, z, t)$ in space and time by solving the second Fick's law in the following form $[25,26]$

$$
\begin{gathered}
\frac{\partial \Phi_{\rho}(x, y, z, t)}{\partial t}=\frac{\partial}{\partial x}\left[D_{\Phi_{\rho}}(x, y, z, T) \frac{\partial \Phi_{\rho}(x, y, z, t)}{\partial x}\right]+\frac{\partial}{\partial y}\left[D_{\Phi_{\rho}}(x, y, z, T) \frac{\partial \Phi_{\rho}(x, y, z, t)}{\partial y}\right]+ \\
+\frac{\partial}{\partial z}\left[D_{\Phi_{\rho}}(x, y, z, T) \frac{\partial \Phi_{\rho}(x, y, z, t)}{\partial z}\right]+\Omega \frac{\partial}{\partial x}\left[\frac{D_{\Phi_{\rho} S}}{k T} \nabla_{s} \mu_{1}(x, y, z, t) \int_{0}^{L_{z}} \Phi_{\rho}(x, y, W, t) d W\right]+ \\
+\Omega \frac{\partial}{\partial y}\left[\frac{D_{\Phi_{\rho} S}}{k T} \nabla_{s} \mu_{1}(x, y, z, t) \int_{0}^{L_{z}} \Phi_{\rho}(x, y, W, t) d W\right]+k_{\rho, \rho}(x, y, z, T) \rho^{2}(x, y, z, t)+ \\
+\frac{\partial}{\partial x}\left[\frac{D_{\Phi_{\rho} S}}{\bar{V} k T} \frac{\partial \mu_{2}(x, y, z, t)}{\partial x}\right]+\frac{\partial}{\partial y}\left[\frac{D_{\Phi_{\rho} S}}{\bar{V} k T} \frac{\partial \mu_{2}(x, y, z, t)}{\partial y}\right]+\frac{\partial}{\partial z}\left[\frac{D_{\Phi_{\rho} s}}{\bar{V} k T} \frac{\partial \mu_{2}(x, y, z, t)}{\partial z}\right]+ \\
+k_{\rho}(x, y, z, T) \rho(x, y, z, t) .
\end{gathered}
$$


Initial and boundary conditions for above equations could be written as

$$
\begin{gathered}
\left.\frac{\partial \Phi_{\rho}(x, y, z, t)}{\partial x}\right|_{x=0}=0,\left.\frac{\partial \Phi_{\rho}(x, y, z, t)}{\partial x}\right|_{x=L_{x}}=0,\left.\frac{\partial \Phi_{\rho}(x, y, z, t)}{\partial y}\right|_{y=0}=0, \\
\left.\frac{\partial \Phi_{\rho}(x, y, z, t)}{\partial y}\right|_{y=L_{y}}=0,\left.\frac{\partial \Phi_{\rho}(x, y, z, t)}{\partial z}\right|_{z=0}=0,\left.\frac{\partial \Phi_{\rho}(x, y, z, t)}{\partial z}\right|_{z=L_{z}}=0, \\
\Phi_{\rho}(x, y, z, 0)=f_{\Phi \rho}(x, y, z) .
\end{gathered}
$$

Functions $D_{\Phi \rho}(x, y, z, T)$ describe dependences of coefficients of volumetric diffusion on spatial coordinates and temperature; functions $D_{\Phi \rho S}(x, y, z, T)$ describe dependences of coefficients of surficial diffusion on spatial coordinates and temperature; functions $k_{\rho}(x, y, z, T)$ describe dependences of parameters of decay of the considered simplest complexes of radiation defects on spatial coordinates and temperature.

Chemical potential $\mu_{1}$, which was considered in the Eq.(1), could be calculated by using the following relation [20]

$$
\mu_{1}=E(z) \Omega \sigma_{i j}\left[u_{i j}(x, y, z, t)+u_{j i}(x, y, z, t)\right] / 2 .
$$

Here function $E(z)$ describes the spatial dependences of Young modulus; values $\sigma_{i j}$ and $u_{i j}=\frac{1}{2}\left(\frac{\partial u_{i}}{\partial x_{j}}+\frac{\partial u_{j}}{\partial x_{i}}\right)$ describe the stress tensor and the deformation tensor, respectively; values $u_{i}, u_{j}$ correspond to components $u_{x}(x, y, z, t), u_{y}(x, y, z, t)$ and $u_{z}(x, y, z, t)$ of the displacement vector $\breve{u}(x, y, z, t)$, which could be obtained due to nonzero lattice distances of layers in the considered heterostructure; parameters $x_{i}, x_{j}$ correspond to spatial coordinate $x, y, z$. The Eq. (7) could be transform to the following form

$$
\begin{aligned}
& \mu(x, y, z, t)=\left[\frac{\partial u_{i}(x, y, z, t)}{\partial x_{j}}+\frac{\partial u_{j}(x, y, z, t)}{\partial x_{i}}\right]\left\{\frac{1}{2}\left[\frac{\partial u_{i}(x, y, z, t)}{\partial x_{j}}+\frac{\partial u_{j}(x, y, z, t)}{\partial x_{i}}\right]-\right. \\
& \left.-\varepsilon_{0} \delta_{i j}+\frac{\sigma(z) \delta_{i j}}{1-2 \sigma(z)}\left[\frac{\partial u_{k}(x, y, z, t)}{\partial x_{k}}-3 \varepsilon_{0}\right]-K(z) \beta(z)\left[T(x, y, z, t)-T_{0}\right] \delta_{i j}\right\} \frac{\Omega}{2} E(z) .
\end{aligned}
$$


Parameter $\sigma$ describes the Poisson coefficient; parameter $\varepsilon_{0}=\left(a_{s}-a_{E L}\right) / a_{E L}$ describes the mismatch parameter, where $a_{E L}$ and $a_{S}$ are the lattice distances of the epitaxial layer and the substrate; value $K$ describes the uniform compression modulus; value $\beta$ describes the thermal expansion coefficient; parameter $T_{r}$ describes the temperature of equilibrium state, which was considered as the room temperature. We calculate components of displacement vector as solution of the following equations [21]

$$
\left\{\begin{array}{l}
\rho(z) \frac{\partial^{2} u_{x}(x, y, z, t)}{\partial t^{2}}=\frac{\partial \sigma_{x x}(x, y, z, t)}{\partial x}+\frac{\partial \sigma_{x y}(x, y, z, t)}{\partial y}+\frac{\partial \sigma_{x z}(x, y, z, t)}{\partial z} \\
\rho(z) \frac{\partial^{2} u_{y}(x, y, z, t)}{\partial t^{2}}=\frac{\partial \sigma_{y x}(x, y, z, t)}{\partial x}+\frac{\partial \sigma_{y y}(x, y, z, t)}{\partial y}+\frac{\partial \sigma_{y z}(x, y, z, t)}{\partial z} \\
\rho(z) \frac{\partial^{2} u_{z}(x, y, z, t)}{\partial t^{2}}=\frac{\partial \sigma_{z x}(x, y, z, t)}{\partial x}+\frac{\partial \sigma_{z y}(x, y, z, t)}{\partial y}+\frac{\partial \sigma_{z z}(x, y, z, t)}{\partial z}
\end{array}\right.
$$

Here $\quad \sigma_{i j}=\frac{E(z)}{2[1+\sigma(z)]}\left[\frac{\partial u_{i}(x, y, z, t)}{\partial x_{j}}+\frac{\partial u_{j}(x, y, z, t)}{\partial x_{i}}-\frac{\delta_{i j}}{3} \frac{\partial u_{k}(x, y, z, t)}{\partial x_{k}}\right]+K(z) \delta_{i j} \times$ $\times \frac{\partial u_{k}(x, y, z, t)}{\partial x_{k}}-\beta(z) K(z)\left[T(x, y, z, t)-T_{r}\right]$; function $\rho(z)$ describes the spatial dependences of materials of heterostructure; parameter $\delta_{i j}$ describes the symbol of Kronecker. Initial and boundary conditions for the recently considered system of equations (8) could be presented as

$$
\begin{aligned}
& \frac{\partial \tilde{u}(0, y, z, t)}{\partial x}=0 ; \frac{\partial \tilde{u}\left(L_{x}, y, z, t\right)}{\partial x}=0 ; \frac{\partial \tilde{u}(x, 0, z, t)}{\partial y}=0 ; \frac{\partial \tilde{u}\left(x, L_{y}, z, t\right)}{\partial y}=0 ; \\
& \frac{\partial \breve{u}(x, y, 0, t)}{\partial z}=0 ; \frac{\partial \breve{u}\left(x, y, L_{z}, t\right)}{\partial z}=0 ; \ddot{u}(x, y, z, 0)=u_{0} ; \ddot{u}(x, y, z, \infty)=\breve{u}_{0} .
\end{aligned}
$$

We calculate distributions of concentrations of considered dopant and radiation defects in space and time by solution the equations (1), (3), (5) and (8) by using approach of averaging of function corrections in the standard form [28]. In the framework of the paper we calculate concentration of infused/implanted dopant; concentrations of radiation defects, which were generated during ion implantation; components of displacement vector, which could be obtained due to nonzero lattice distances of 
layers in the considered heterostructure. We calculate the considered function by using the second-order approximation in the framework of the considered method of solution. The considered approximation recently has enough large order for qualitative analysis of considered processes and for obtaining of several quantitative results. All analytically calculated functions were checked by their comparison with results of direct numerical simulations.

\section{Discussion}

In this part of the paper we analyzed how distribution of concentration of dopant was changed with time, variation of other parameters, with account transformation of concentrations of radiation defects (both point radiation defects and their simplest complexes) and components of displacement vector with account modification of porosity. Figures 2 and 3 presents some typical spatial distributions of dopant concentrations. Distributions on figure 2 corresponds to infusion of dopant. Distributions on figure 3 corresponds to implanted of dopant. Diffusion coefficient of dopant in doped area is larger in comparison with diffusion coefficient of dopant in undoped area. From the considered figures one can find, that distributions of dopant concentrations could be more compact in heterostructures in comparison with analogous distributions in homogenous materials. At the same time on can find increasing of homogeneity of the considered distributions in doped area. It should be noted, that the considered approach for increasing compactness and homogeneity of distribution of concentration of dopant leads to necessity of optimization of annealing of the considered dopant and radiation defects (after ion implantation) to obtain compromise between increasing of the considered compactness and considered homogeneity of distribution of concentration of considered dopant. To obtain the compromise we used the recently introduced approach [29-37]. To use the approach one shall approximate real distribution of dopant's concentration by a step-wise function, which illustrates requite ideal distribution of dopant's concentration. The step-wise approximation function with real distributions of dopant are presented on figures 4 and 5. To choose real distributions of dopant, which corresponds to minimal error between the distribution and the considered approximation function, one shall to choose appropriate annealing time. 
To choose the annealing time one shall minimize mean-squared error

$$
U=\frac{1}{L_{x} L_{y} L_{z}} \int_{0}^{L_{x}} \int_{0}^{L_{y} L_{z}}\left[\int_{0}[C(x, y, z, \Theta)-\psi(x, y, z)] d z d y d x .\right.
$$

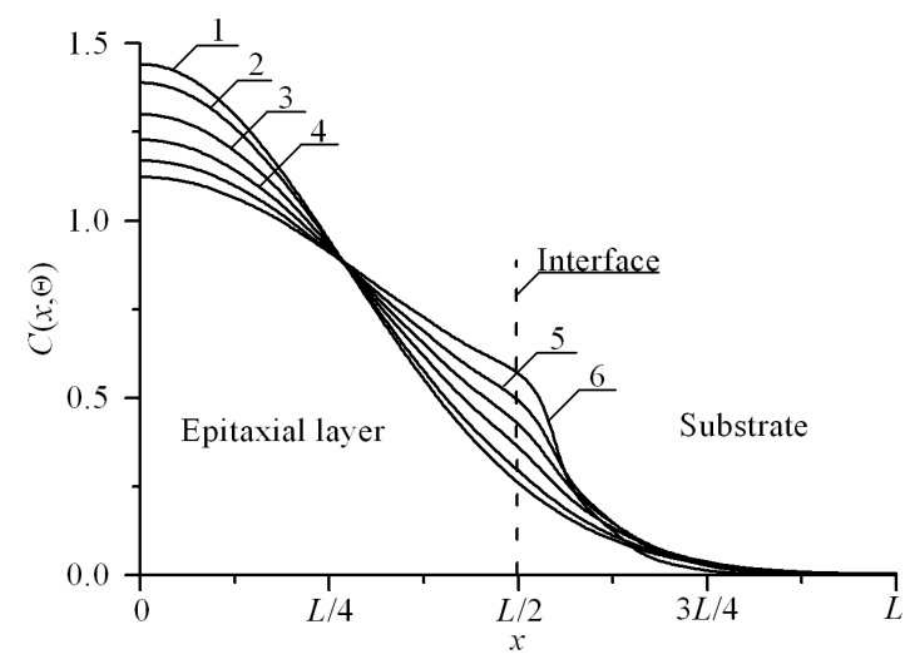

Fig. 2. Distributions of infused dopant concentration. Increasing of difference between values of diffusion coefficient of dopant correspond to increasing of number of

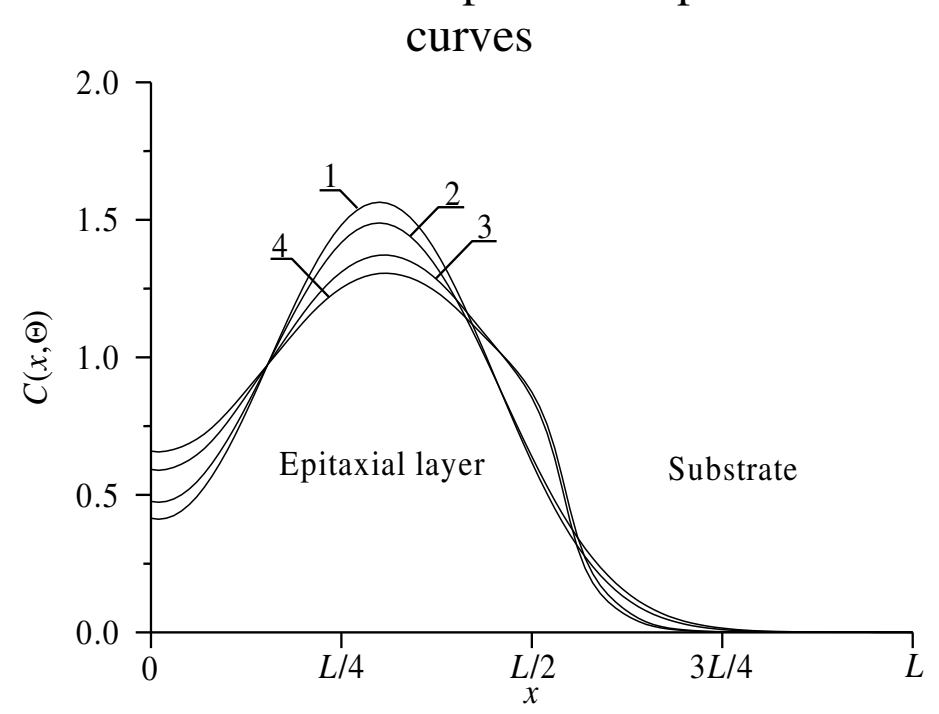

Fig. 3. Distributions of infused dopant concentration. Increasing of difference between values of diffusion coefficient of dopant correspond to increasing of number of curves. Annealing time is equal to $\Theta=0.0048\left(L_{x}{ }^{2}+L_{y}{ }^{2}+L_{z}{ }^{2}\right) / D_{0}$ for curves 1 and 3. Annealing time is equal to $\Theta=0.0057\left(L_{x}^{2}+L_{y}{ }^{2}+L_{z}{ }^{2}\right) / D_{0}$ for curves 2 and 4 


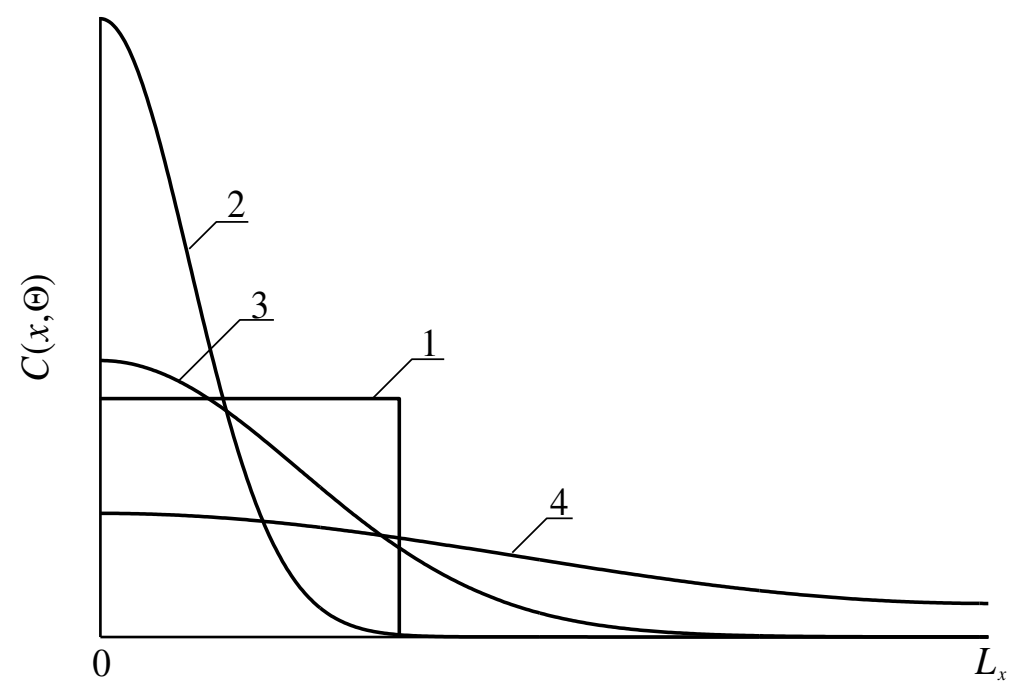

Fig. 4. Distributions of concentrations of infused dopant. Curve 1 describes step-wise approximation function. Curves 2-4 describe real distributions of concentration of infused dopant. Increasing of annealing time corresponds to increasing of curve's number Here function $\psi(x, y, z)$ describes the considered step-wise approximation function. Several dependences of optimal values of the considered annealing time on several parameters were presented on figures 6 (for infused dopant) and 7 (for implanted dopant). It is known, that using ion implantation leads to necessity organize radiation defects annealing. It is also known, that radiation defects annealing leads to spreading of distribution of dopant concentration. Ideal annealing of defects corresponds to achievement the nearest interfaces between materials of heterostructure by the implanted dopant. If the considered dopant can not achieve the required interface, it is attracted an interest to make additional annealing of the implanted dopant. In the framework of this situation optimal values of the considered additional annealing time of implanted dopant became smaller in comparison with annealing time of infused dopant. 


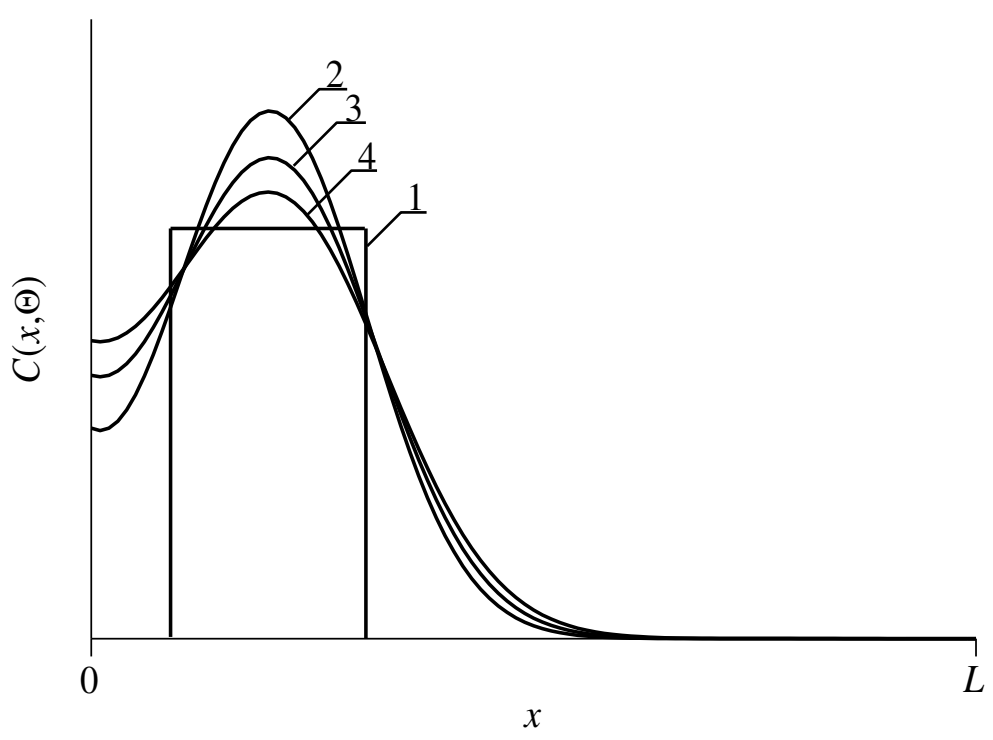

Fig. 5. Distributions of concentrations of implanted dopant. Curve 1 describes step-wise approximation function. Curves 2-4 describe real distributions of concentration of infused dopant. Increasing of annealing time corresponds to increasing of curve's number

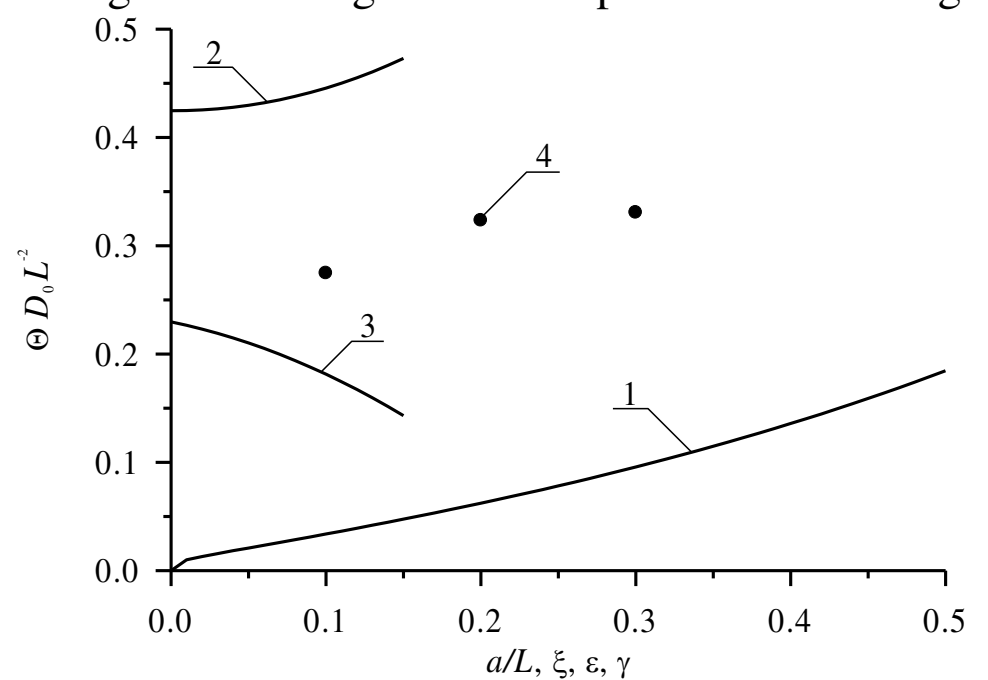

Fig. 6. Dependences of the optimized annealing time of infused dopant. Curve 1 describes dependence of the considered time on the relation $a / L$. Curve 2 describes dependence of the considered time on the parameter $\varepsilon$. Curve 3 describes dependence of the considered time on the parameter $\xi$. Curve 4 describes dependence of the considered time on the parameter $\gamma$

Now let us analyzed modification of dopant's concentration distribution under influence of missmatch-induced stress. One can consider compression of the considered distributions at $\varepsilon_{0}<0$. At the same time one can consider spreading of the considered distributions at $\varepsilon_{0}>0$. The obtained transformation of the considered concentration of dopant could be partially compensated due to annealing by laser [37]. The considered type of annealing of dopant leads to acceleration of dopant diffusion and other processes by generation of inhomogenous distribution of annealing temperature. Mis- 
match-induced stress leads to changing of optimized annealing time, but the stress decreases by porosity of materials. To illustrate the decreasing figure 8 shows displacement vector component in porous and nonporous materials and figure 9 shows distributions of concentration of vacancies in porous and nonporous materials.

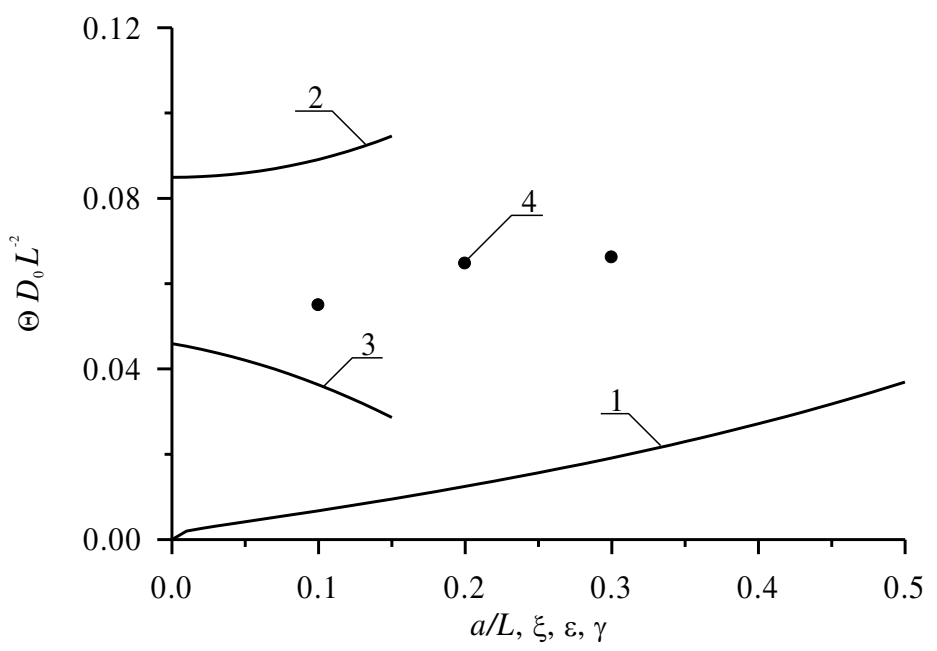

Fig. 7. Dependences of the optimized annealing time of implanted dopant. Curve 1 describes dependence of the considered time on the relation $a / L$. Curve 2 describes dependence of the considered time on the parameter $\varepsilon$. Curve 3 describes dependence of the considered time on the parameter $\xi$. Curve 4 describes dependence of the con-

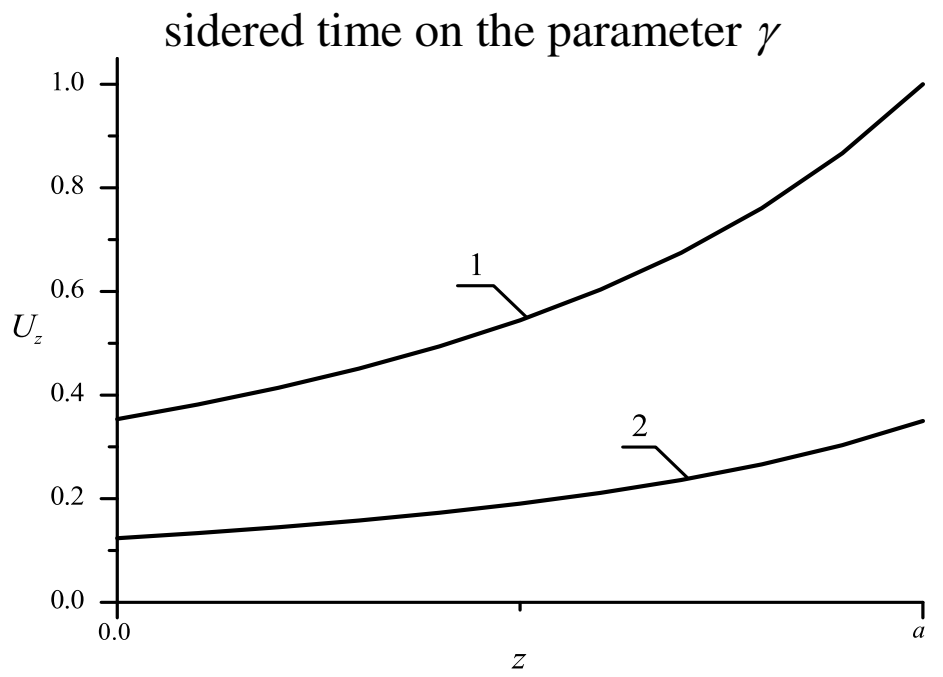

Fig. 8. Dependences of displacement vector component $u_{z}$ after normalization. Curve 1 describes the considered component $u_{z}$ in nonporous material. Curve 2 describes the considered component $u_{z}$ in porous material 


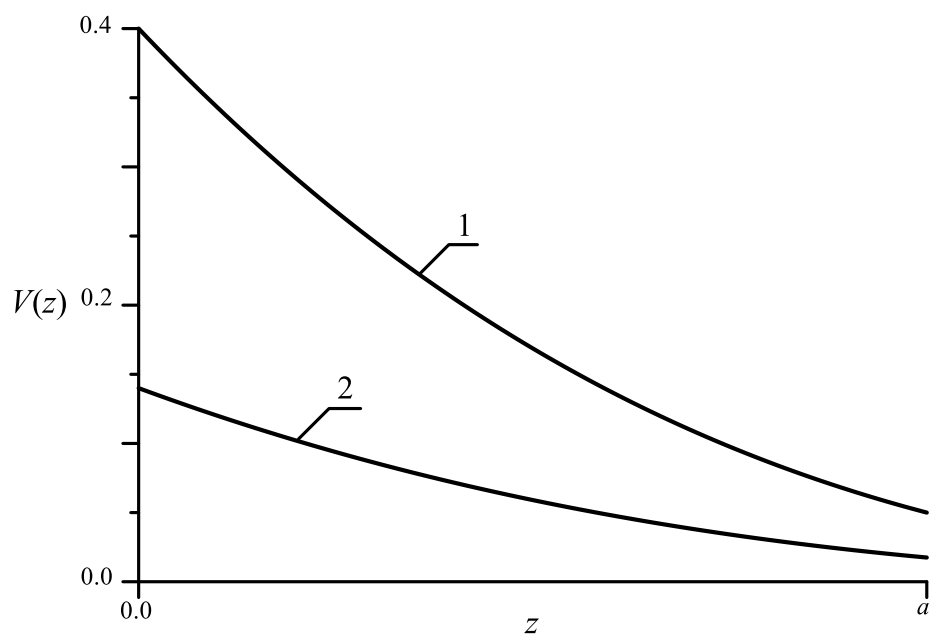

Fig. 9. Distribution of concentration of vacancies on coordinate $z$. Curve 1 describes the considered distribution in unstressed material. Curve 2 describes the considered distribution in stressed material

\section{Conclusion}

We analyzed modification of dopant after infusion and implantation into a heterostructure to manufacture field-effect transistors in the framework of a four stages distributed amplifier. Recommendations for reduction of dimensions of transistors in the framework of the considered amplifier and mismatch-induced stress were formulated. We introduce an analytical approach for prognosis of diffusion and ion types of doping with account changing of physical and technological parameters in space and time was introduced. At the same time the introduced approach leads to possibility to take into account nonlinearity of considered processes.

\section{References}

[1] V.I. Lachin, N.S. Savelov. Electronics. Rostov-on-Don: Phoenix, 2001.

[2] A. Polishscuk. Modern Electronics. Issue 12. P. 8-11 (2004).

[3] G. Volovich. Modern Electronics. Issue 2. P. 10-17 (2006).

[4] A. Kerentsev, V. Lanin, Power Electronics. Issue 1. P. 34 (2008).

[5] A.O. Ageev, A.E. Belyaev, N.S. Boltovets, V.N. Ivanov, R.V. Konakova, Ya.Ya. Kudrik, P.M. Litvin, V.V. Milenin, A.V. Sachenko. Semiconductors. Vol. 43 (7). P. 897-903 (2009).

[6] Jung-Hui Tsai, Shao-Yen Chiu, Wen-Shiung Lour, Der-Feng Guo. Semiconductors.

Vol. 43 (7). C. 971-974 (2009). 
[7] O.V. Alexandrov, A.O. Zakhar'in, N.A. Sobolev, E.I. Shek, M.M. Makoviychuk, E.O. Parshin.Semiconductors. Vol. 32 (9). P. 1029-1032 (1998).

[8] I.B. Ermolovich, V.V. Milenin, R.A. Red'ko, S.M. Red'ko. Semiconductors. Vol. 43 (8). P. 1016-1020 (2009).

[9] P. Sinsermsuksakul, K. Hartman, S.B. Kim, J. Heo, L. Sun, H.H. Park, R. Chakraborty, T. Buonassisi, R.G. Gordon. Appl. Phys. Lett. Vol. 102 (5). P. 053901053905 (2013).

[10] J.G. Reynolds, C.L. Reynolds, Jr.A. Mohanta, J.F. Muth, J.E. Rowe, H.O. Everitt, D.E. Aspnes. Appl. Phys. Lett. Vol. 102 (15). P. 152114-152118 (2013).

[11] N.I. Volokobinskaya, I.N. Komarov, T.V. Matyukhina, V.I. Reshetnikov, A.A. Rush, I.V. Falina, A.S. Yastrebov. Semiconductors. Vol. 35 (8). P. 1013-1017 (2001).

[12] E.L. Pankratov, E.A. Bulaeva. Reviews in Theoretical Science. Vol. 1 (1). P. 5882 (2013).

[13] S.A. Kukushkin, A.V. Osipov, A.I. Romanychev. Physics of the Solid State. Vol. 58 (7). P. 1448-1452 (2016).

[14] E.M. Trukhanov, A.V. Kolesnikov, I. D. Loshkarev. Russian Microelectronics. Vol. 44 (8). P. $552-558$ (2015).

[15] E.L. Pankratov, E.A. Bulaeva. Reviews in Theoretical Science. Vol. 3 (4). P. 365-398 (2015).

[16] K.K. Ong, K.L. Pey, P.S. Lee, A.T.S. Wee, X.C. Wang, Y.F. Chong. Appl. Phys. Lett. Vol. 89 (17). P. 172111-172114 (2006).

[17] H.T. Wang, L.S. Tan, E. F. Chor. J. Appl. Phys. Vol. 98 (9). P. 094901-094905 (2006).

[18] Yu.V. Bykov, A.G. Yeremeev, N.A. Zharova, I.V. Plotnikov, K.I. Rybakov, M.N. Drozdov, Yu.N. Drozdov, V.D. Skupov. Radiophysics and Quantum Electronics. Vol. 43 (3). P. 836-843 (2003).

[19] J. Abu-Taha, M. Yazg. Analog. Integr. Circ. Sig. Process. Vol. 86. P. 429-438 (2016).

[20] Y.W. Zhang, A.F. Bower. Journal of the Mechanics and Physics of Solids. Vol. 47 (11). P. 2273-2297 (1999). 
[21] L.D. Landau, E.M. Lefshits. Theoretical physics. 7 (Theory of elasticity) (Physmatlit, Moscow, 2001).

[22] M. Kitayama, T. Narushima, W.C. Carter, R.M. Cannon, A.M. Glaeser. J. Am. Ceram. Soc. Vol. 83. P. 2561 (2000); M. Kitayama, T. Narushima, A.M. Glaeser. J. Am. Ceram. Soc. Vol. 83. P. 2572 (2000).

[23] P.G. Cheremskoy, V.V. Slesov, V.I. Betekhtin. Pore in solid bodies (Energoatomizdat, Moscow, 1990).

[24] Z.Yu. Gotra, Technology of microelectronic devices (Radio and communication, Moscow, 1991).

[25] P.M. Fahey, P.B. Griffin, J.D. Plummer. Rev. Mod. Phys. Vol. 61 (2). P. 289 388 (1989).

[26] V.L. Vinetskiy, G.A. Kholodar', Radiative physics of semiconductors. ("Naukova Dumka", Kiev, 1979).

[27] M.G. Mynbaeva, E.N. Mokhov, A.A. Lavrent'ev, K.D. Mynbaev, Techn. Phys. Lett. Vol. 34 (17). P. 13 (2008).

[28] Yu.D. Sokolov. Applied Mechanics. Vol. 1 (1). P. 23-35 (1955).

[29] E.L. Pankratov. Russian Microelectronics. Vol. 36 (1). P. 33-39 (2007).

[30] E.L. Pankratov. Int. J. Nanoscience. Vol. 7 (4-5). P. 187-197 (2008).

[31] E.L. Pankratov, E.A. Bulaeva. Reviews in Theoretical Science. Vol. 1 (1). P. 5882 (2013).

[32] E.L. Pankratov, E.A. Bulaeva. Int. J. Micro-Nano Scale Transp. Vol. 3 (3). P. 119-130 (2012).

[33] E.L. Pankratov, E.A. Bulaeva. International Journal of Modern Physics B. Vol. 29 (5). P. 1550023-1-1550023-12 (2015).

[34] E.L. Pankratov. J. Comp. Theor. Nanoscience. Vol. 14 (10). P. $4885-4899$ (2017).

[35] E.L. Pankratov, E.A. Bulaeva. Materials science in semiconductor processing. Vol. 34. P. 260-268 (2015).

[36] E.L. Pankratov, E.A. Bulaeva. Int. J. Micro-Nano Scale Transp. Vol. 4 (1). P. 17 31 (2014).

[37] E.L. Pankratov. Nano. Vol. 6 (1). P. 31-40 (2011). 